\title{
Pain perception and dental anxiety during periodontal probing in patients visiting community oral health programme: a cross sectional study
}

\author{
Abanish Singh ${ }^{*^{*}}$, Ashish Shrestha ${ }^{2}$ (B) and Tarakant Bhagat ${ }^{2}$ (1)
}

\begin{abstract}
Background: Periodontal probing is one of the basic clinical oral examination procedures. It is carried out to assess the severity of gingival and periodontal disease. The experience of pain during probing may discourage patients. So, this study was conducted to estimate the pain perception and dental anxiety experienced during periodontal probing in patients visiting the community oral health programmes of B. P. Koirala Institute of Health Sciences (BPKIHS).

Methods: A cross-sectional study was conducted among 100 participants of community oral health programmes of BPKIHS. Demographic profile, WHO modified Community Periodontal Index (CPI) 2013, Pain perception via Visual Analogue Scale (VAS Scores) and Short Version of Spielberger State-Trait Anxiety Inventory (STAI) Self-evaluation Questionnaire (Y-6 item) were assessed. Mean \pm SD and Spearman correlation for pain and anxiety were computed.

Results: Only $10 \%$ of the study participants had healthy gingiva and $12 \%$ had periodontal pockets. Pain perception and dental anxiety was present in the participants. The participants experienced very little pain $(6.75 \pm 10.65)$ during periodontal probing. The overall anxiety score was $13.37 \pm 1.81$. There was a very weak correlation between the VAS Scores and the anxiety scores of the participants.

Conclusion: This study concludes that pain perception and anxiety are low during periodontal probing. There was no correlation between bleeding on probing with pain and anxiety among the people visiting community oral health programmes of BPKIHS.
\end{abstract}

Keywords: Community oral health programme, Dental anxiety, Pain perception, Periodontal probing

\section{Background}

International Association for the study of Pain (IASP) defines pain as "an unpleasant sensory and emotional experience associated with actual or potential tissue damage, or described in terms of such damage" [1]. Pain cannot simply be determined by the intensity of nociceptive stimulation [2]. Pain not only is a physiological

\footnotetext{
*Correspondence: dr.abanishsingh@gmail.com

${ }^{1}$ Narayani Hospital, Birgunj, Parsa, Nepal

Full list of author information is available at the end of the article
}

experience; rather includes cognitive and emotional construct [3]. Periodontal probe is a commonly used instrument to assess periodontal conditions and the severity of periodontal lesions $[4,5]$. However, patient discomfort and pain associated with the insertion of a periodontal probe into the periodontal pocket are common clinical events [6].

Periodontal probing is used to measure clinical parameters like bleeding on probing, probing depth, clinical attachment level (CAL) and so on. This gives us an idea regarding the disease severity of periodontal structure. 
The intensity of pain or discomfort has been perceived by practitioners to differ dramatically between patients [6]. The experiences of pain during probing and scaling may also discourage patients who do not have periodontitis. The individual characteristics such as age, smoking and oral health status effects patients' pain perception and dental anxiety. An unpleasant dental experience has a strong impact on dental anxiety. Extreme pain experience after dental work is amongst the most common distressing life experiences, and has seen to trigger psychological trauma and a persistent fear of the dentist among patients [7].

Oral health education via different media changes attitude and practice as well as improves oral hygiene habits, oral health awareness and knowledge level [8]. Oral health education and promotion should be done to overcome the dental fear and anxiety [9]. The prevalence of deep periodontal pocket is around 31\% in Nepal [10]. There is a paucity of research conducted to assess the pain perception and dental anxiety during periodontal probing and no research has been conducted in Nepal addressing the pain perception of the patient in this context. With an assumption that the pain perception and dental anxiety increases with periodontal probing, this study aims to assess the pain perception and dental anxiety during periodontal probing in patients visiting the community oral health programmes of B. P. Koirala Institute of Health Sciences (BPKIHS), Dharan, Nepal.

\section{Methods}

A cross-sectional study was conducted among 100 patients visiting community oral health programmes of BPKIHS. The study was conducted from February 2018 to July 2018. Ethical approval for the study was obtained from the Institutional Review Committee, BPKIHS, Dharan (Ref. No: IRC/1033/017). The study adhered to STROBE guidelines. The community oral health programmes of BPKIHS is regularly conducted on weekly basis at Maternal and Child Health Care Center, Itahari, Nepal government Health Post at Bhedetar, Chatara and Tarhara. Adult Patients (18-75 years of age) who visited the Community Oral Health Programs of BPKIHS were included in the study (Fig. 1). We excluded the patients requiring prophylactic antibiotics before probing, patients suffering from mental disorders or with chronic pain problems, patients having previous experience of painful dental visit, patients suffering from coagulation/ bleeding disorders, pregnant or lactating mothers, patients under antidepressant and analgesic medications, patients having acute periodontal pain (with abscess, pulpitis or acute infections). This study considered (95\% CI) to estimate the sample size. For this purpose, mean \pm SD $(19.1 \pm 9.6)$ value was taken from the study [11] done by

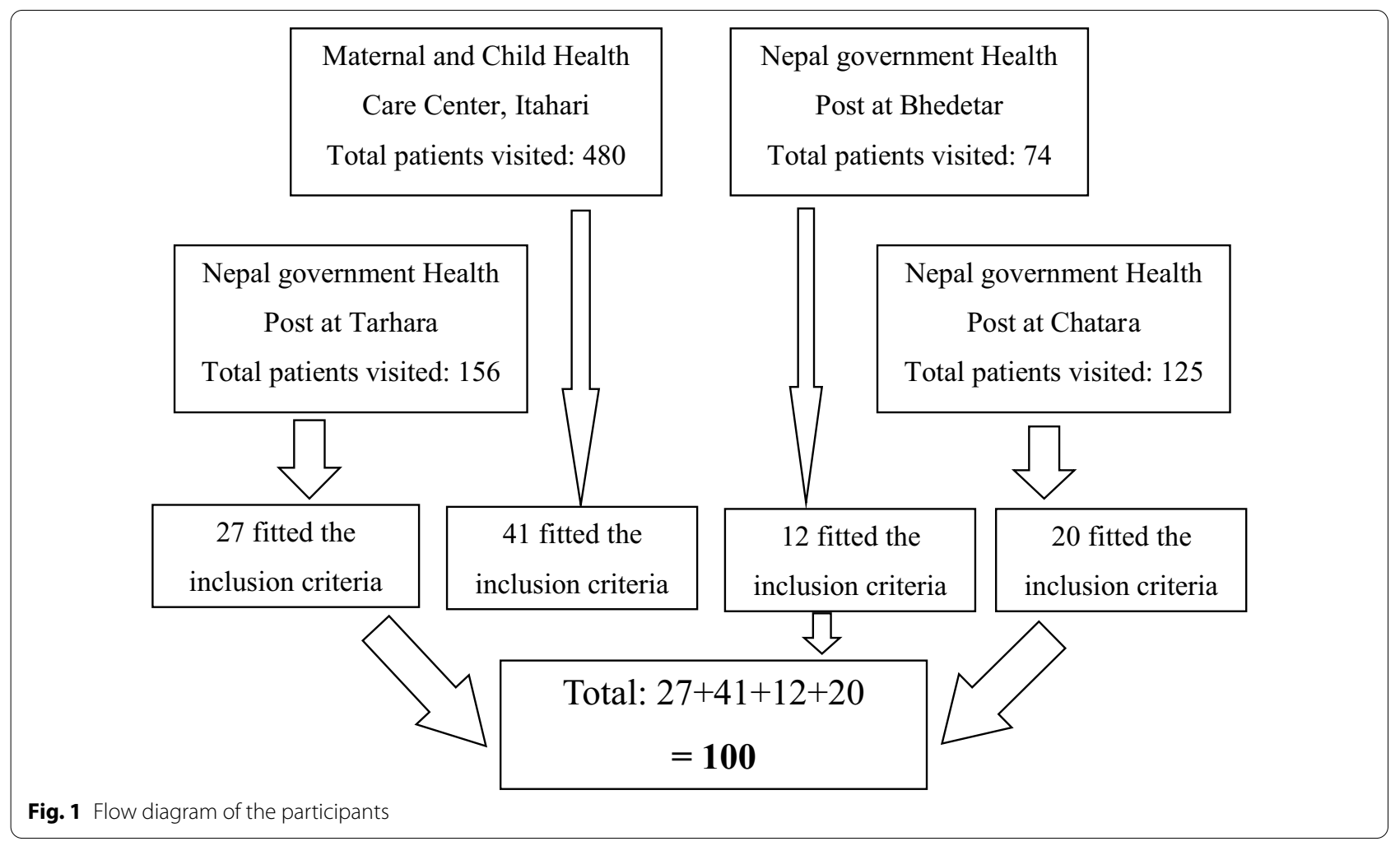


Canakci and Canakci. A convenience sampling method was used. All patients who visited the community oral health programmes and those satisfying inclusion and exclusion criteria were enrolled into the study.

\section{Survey instruments}

- Questionnaire for accessing oral hygiene practices and demographic profile: The questionnaires were translated in Nepali followed by face and content validation by expert faculties.

- Visual Analogue Scale (VAS) [12]: It is most common method used in epidemiological studies to access pain perception. It is simple, reliable and most accepted method. The scale was anchored by "no pain" (score 0) and "pain as bad as it could be" or worst imaginable pain" (score of 100 [100-mm scale])

- Short Version of Spielberger State-Trait Anxiety Inventory (STAI) Self-evaluation Questionnaire (Y-6 item) [13]: It is a six item questionnaire that rate the anxiety on a likert-scale as 1: not at all anxious, 2 : somewhat anxious, 3 : moderately anxious and 4 : very much anxious.

- Modified Community Periodontal Index as that of WHO Oral Health Survey Methods 2013 [14]: It scores the presence and absence of gingival bleeding and periodontal pocket of individual tooth.

Procedures and schedules Informed Consent was taken from all the patients. All the participants were examined by a trained and calibrated examiner, seated in a chair, under the natural light with a plane mouth mirror and WHO periodontal probe. Oral examination was followed by recording of the Modified Community Periodontal Index. Pain perception was assessed using Visual Analogue Scale for each quadrant. All the teeth in a quadrant were probed and participants were asked to rate their pain experience on VAS. Subsequently, the participants were asked to encircle the scores of the Short Version of Spielberger State-Trait Anxiety Inventory (STAI) Self-evaluation Questionnaire (Y-6 item) for assessing their anxiety experienced during periodontal probing. People diagnosed with dental caries, periodontal diseases and any other oral lesions were treated as per feasibility and resources available at the community field programs (like Atraumatic restorative Treatment and Extractions). Others were referred to the Department of Public Health Dentistry, College of Dental Surgery, B. P. Koirala Institute of Health Sciences, Dharan for needful treatment. A pilot examination was conducted to validate the entire questionnaire (pro forma) that was translated in Nepali version. The translation was done as per WHO oral health survey methods 2013 guidelines. The pilot study was conducted among 10 participants prior to the main study and these participants were not included in the main study. The Cronbach alpha value for the anxiety questionnaires and intra-class correlation coefficient for VAS Scores were found to be 0.76 (acceptable reliability) and 0.81 (good reliability) respectively.

\section{Statistical analysis}

After completion of the survey, data obtained were entered in Microsoft Excel Sheet 2007 and statistical analysis was done in Statistical Package for Social Sciences (SPSS) version 11.5 (SPSS, Inc., Chicago, IL, USA) software. Descriptive statistics including the mean and standard deviations were computed for VAS Score, anxiety score and CPI Score. Inferential Statistics: included; correlation between mean VAS Scores for the maxillary and mandibular arch obtained using Spearman Correlation Coefficient; and Mean VAS score and anxiety score compared based on gender using Kruskal-Wallis test.

\section{Results}

Out of 100 participants, majorities were male (57\%). Almost all (98\%) of the participants were found brushing their teeth. About $80 \%$ were seen to brush for 2 to $5 \mathrm{~min}$ (Table 1).

\section{Table 1 Demographic characteristics}

\begin{tabular}{ll}
\hline Demographic characteristics & Percentage \\
\hline Gender & 57 \\
Male & 43 \\
Female & \\
Marital status & 51 \\
Married & 49 \\
Unmarried & \\
Socioeconomic status & 37 \\
Lower class & 63 \\
Middle class & 0 \\
Upper class & \\
Time taken for brushing & 14 \\
1-2 min & 80 \\
2-5 min & 6 \\
More than 5 min & \\
Type of tooth brush & 13 \\
Hard & 5 \\
Medium & 19 \\
Soft & 62 \\
Don't know & \\
Type of toothpaste & 39 \\
Fluoridated & 61 \\
Non-fluoridated/don't know &
\end{tabular}


Table 2 Community Periodontal Index (WHO modified 2013)

\begin{tabular}{|c|c|c|c|c|c|}
\hline \multicolumn{3}{|l|}{ Gingival status } & \multicolumn{3}{|l|}{ Periodontal status } \\
\hline & Scores & Percentage & & Scores & Percentage \\
\hline Absence of condition & 0 & 10 & Absence of condition & 0 & 41 \\
\hline Presence of condition & 1 & 72 & Pocket $4-5$ mm & 1 & 11 \\
\hline Teeth excluded & 9 & 0 & Pocket 6 mm or, more & 2 & 1 \\
\hline Tooth not present & $x$ & 47 & Tooth excluded & 9 & 0 \\
\hline
\end{tabular}

Table 3 Mean \pm SD visual analogue scale score

\begin{tabular}{llcl}
\hline Quadrant & \multicolumn{3}{l}{ Mean \pm SD } \\
\cline { 2 - 4 } & Male & Female & Total \\
\hline 1 & $4.88 \pm 8.79$ & $3.74 \pm 7.83$ & $4.39 \pm 8.35$ \\
2 & $5.98 \pm 11.04$ & $4.16 \pm 9.47$ & $5.20 \pm 10.38$ \\
3 & $5.43 \pm 9.01$ & $10.60 \pm 15.12$ & $9.46 \pm 14.46$ \\
4 & $8.60 \pm 13.98$ & $7.35 \pm 12.27$ & $7.98 \pm 15.05$ \\
Overall & $6.97 \pm 11.53$ & $6.46 \pm 9.48$ & $6.75 \pm 10.65$ \\
\hline
\end{tabular}

SD Standard deviation

Table 4 Comparison of VAS score and anxiety score between age groups

\begin{tabular}{llcclc}
\hline & Age group & $\mathbf{n}$ & Mean \pm SD & Mean rank & $P$-value \\
\hline Anxiety & $18-40$ years & 85 & $13.54 \pm 1.72$ & 51.01 & 0.669 \\
& $41-75$ years & 15 & $13.07 \pm 2.28$ & 47.63 & \\
VAS score & $18-40$ years & 85 & $6.00 \pm 9.50$ & 48.08 & $0.038^{*}$ \\
& $41-75$ years & 15 & $11.05 \pm 15.41$ & 64.23 & \\
\hline
\end{tabular}

SD Standard deviation

*Kruskal-Wallis test

Only $10 \%$ of the study populations had healthy gingival. Twelve participants were found to have periodontal pockets of which one had a deep pocket of $6 \mathrm{~mm}$ or more (Table 2).

Overall mean VAS score of the study participant was $6.75 \pm 10.65$ where males and females had similar VAS Scores $(6.97 \pm 11.53$ and $6.46 \pm 9.48$ respectively). The difference in the VAS score among males and females was not significant $(p=0.780)$ (Table 3$)$.

The mean age of male participants was $29.7 \pm 11.8$ years and that of female participants was $31.9 \pm 10.6$ years. All the participants were further divided into two categories based on age (less than 40 years and more than 40 years) to see the pain difference in young and old age groups. There was a statistically significant difference in VAS scores between both the age groups with old age participants having higher pain perception $(p=0.038)$. There was no statistically
Table 5 Correlation between VAS Scores between maxilla and mandible

\begin{tabular}{lllrll}
\hline & Median & Mean & SD & Correlation coefficient & $P$-value \\
\hline Maxilla & 0.00 & 4.79 & 8.70 & 0.643 & $<0.001^{*}$ \\
Mandible & 0.50 & 8.72 & 13.75 & & \\
Overall & 2.50 & 6.75 & 10.65 & & \\
\hline \multicolumn{7}{l}{ SD Standard deviation } \\
*Spearman correlation coefficient
\end{tabular}

Table 6 Mean \pm SD of anxiety scores

\begin{tabular}{lcc}
\hline & Mean \pm SD & $P$-value \\
\hline Male & $13.60 \pm 2.03$ & $0.408^{*}$ \\
Female & $13.30 \pm 1.48$ & \\
Overall & $13.47 \pm 1.81$ & \\
\hline
\end{tabular}

SD Standard deviation

*Kruskal-Wallis test

Table 7 Correlation between bleeding on probing with pain and anxiety

\begin{tabular}{llll}
\hline Correlation & Anxiety & Pain (VAS score) \\
\hline Bleeding on probing & $\begin{array}{c}\text { Correlation coef- } \\
\text { ficient* }\end{array}$ & 0.033 & $0.350^{* *}$ \\
& Sig. (2-tailed) & 0.742 & 0.000 \\
N & & 100 & 100 \\
\hline
\end{tabular}

*Spearman correlation coefficient

${ }^{* *}$ Correlation is significant at the 0.01 level (2-tailed)

significant difference in the anxiety scores among both the age groups $(p=0.669)$ (Table 4$)$.

There was a strong and statistically significant correlation of VAS scores between maxilla and mandible $(p<0.001)$ Table 5.

The dental anxiety was also similar in both males $(13.60 \pm 2.03)$ and females $(13.30 \pm 1.48)$ with overall anxiety score $13.37 \pm 1.81$ (Table 6 ).

There was a weak significant correlation between VAS score and bleeding on probing whereas weak or no correlation between anxiety and bleeding on probing (Table 7). 
Table 8 Correlation between VAS score and anxiety scores

\begin{tabular}{lll}
\hline & Anxiety & VAS score \\
\hline Correlation coefficient & 1.000 & $0.103^{*}$ \\
Sig. (2-tailed) & & 0.310 \\
N & 100 & 100
\end{tabular}

*Spearman correlation coefficient

There was a very weak correlation between the VAS Scores and the anxiety scores of the patient (Table 8).

\section{Discussion}

This study provided information about pain perception and dental anxiety during periodontal probing. Oral examination was conducted in oral health outreach programmes, on a chair in a sitting position under natural light. The findings revealed, $72 \%$ of the participants had pathological gingival conditions but very few had periodontal pocket (12\%).

Pain perception of the patient cannot be directly assessed by the dentists as communication skills, individual psychological status and, social and cultural backgrounds of the patient affect the expression of pain experienced. In this study, the participants experienced very less pain $(6.75 \pm 10.65)$ during periodontal probing but the VAS scores were highly variable (0 to 59.5 ). Similar results were obtained in other studies [1, 2, 7]. This might be attributed to the reason that pain measurement is subjective and individual, and the assessment and screening are more difficult because of its physical and psychological properties [15]. Additionally, pain perception is influenced by the patients' systemic conditions, oral pathological status, and patients reporting with the complaint of pain $[6,15]$.

Pain perception of female participants was similar to the male participants $(6.46 \pm 9.48$ and $6.97 \pm 11.53$ respectively), and the difference was not statistically significant $(p=0.780)$. This was similar to the study conducted by Canakci and Canakci [11] but different than the results of Faisal et al. [16]. In general, the clinical impression is that elderly people are usually more tolerant of pain. Nociceptors are lost due to aging [17]. In contrast, this study showed a higher VAS score in the elderly (11.05 \pm 15.41$)$ compared to young participants $(6.00 \pm 9.50)$. This might be due to the reason that pain varies subjectively and is also dependent upon many underlying causes that might be unnoticed clinically. There was a significantly significant correlation between the VAS score of maxilla and mandible. The correlation between bleeding and VAS score was also significant $(p<0.001)$. Bleeding on probing indicated the inflammatory condition of the gingiva that raises the possibility of increased pain perception [5].

Female patients are more anxious than male patients. It may be due to the difference in pain threshold between genders. In contrast, this study revealed that the dental anxiety was also similar in both males $(13.60 \pm 2.03)$ and females $(13.30 \pm 1.48)$ with overall anxiety score $13.37 \pm 1.81$. The anxiety of female participants was less compared to the male participants $(13.30 \pm 1.48$ and $13.60 \pm 2.03$ respectively), but the difference was not statistically significant $(p=0.408)$. The insignificant higher anxiety score in male participants might be attributed to difference in sample participants (male $=57$; female $=43$ ). The higher mean age of the female participants $(31.9 \pm 10.6)$ might have also attributed to insignificant less anxiety score among female. It has been seen that older individuals experience lesser anxiety than their younger counterparts due to general decline in anxiety and many more exposure to diseases and their treatments [18]. This was similar to a study conducted by Shaikh and Kamal [19] and Ghazaleh et al. [20] but different than the results of Faisal et al. [16].

Anxiety is thought to increase pain perception and vice versa [21]. Female is supposed to have more fear compared to the male [21]. In contrast, in this study the anxiety scores for males and females were comparable. There was a very weak correlation between anxiety score and bleeding on probing and anxiety and pain perception. This might be due to the fact that anxiety has an influence on expected pain, but not on the experienced pain [22].

The sampling covered a large population area including four districts of eastern Nepal. This study is the first of its kind done in the population. Hence, it is an added asset for the dentist to have an insight into the pain perception and dental anxiety during periodontal probing and further plan the approach for patient management.

The sampling technique used was convenience sampling that limits the generalizability of the study and gives a scope for selection bias. Moreover, the probing was performed by the WHO probe that increases the chance for subjective variation of force applied during probing. A digital probe would have been better to maintain the constant force of probing. The other limitation being chances of variation in pain response by the patients as full mouth probing was done and there were partially edentulous patients. Measuring anxiety accurately is extremely difficult and therefore it may alter research outcomes. As pain and anxiety are subjective measures, it is difficult to quantify them. A participant may express pain and anxiety to one aspect of examination but not in another. Hence, experimental 
studies with digital monitoring of the probing force will further elaborate regarding the correlation between pain and anxiety.

\section{Conclusion}

This study found low pain perception and anxiety during periodontal probing and there was a very low correlation between bleeding on probing with pain and anxiety among the people visiting community oral health programmes of BPKIHS.

\section{Abbreviations}

BPKIHS: B. P. Koirala Institute of Health Sciences; WHO: World Health Organization; ART: Atraumatic restorative treatment.

\section{Acknowledgements}

We thank Dr. Santosh Kumari Agrawal, Dr. Ujwal Gautam and Mr. Raj Kumar Subedi for their help in formatting and editing the manuscript.

\section{Authors' contributions}

$\mathrm{AS}^{*}$ designed the study, collected and analysed the data and drafted the manuscript. AS and TKB contributed in designing the study and drafting the manuscript. All authors reas and approved the final manuscript. ( ${ }^{*}$ Principal author)

\section{Funding}

None.

\section{Availability of data and materials}

The data supporting the findings of this article are available from the corresponding author.

\section{Ethics approval and consent to participate}

Ethical approval was obtained from Institutional review committee, B. P. Koirala Institute of Health Sciences (BPKIHS), Dharan, Nepal (Committee's Ref. No: IRC/1033/017) and written informed consent were taken from the patients.

\section{Consent for publication}

Data collection was anonymous. No images or other personal details of participants are presented here. The patients were informed about the details collected from them and consent was taken for the use of their details in a scientific publication.

\section{Competing interests}

The authors declare that they have no competing interests.

\section{Author details}

${ }^{1}$ Narayani Hospital, Birgunj, Parsa, Nepal. ${ }^{2}$ Department of Public Health Dentistry, B. P. Koirala Institute of Health Sciences, Dharan, Nepal.

Received: 24 April 2020 Accepted: 11 February 2021

Published online: 23 February 2021

\section{References}

1. Okeson JP. Bell's orofacial pains: the clinical management of orofacial pain. Chicago: Quintessence Publishing Company; 2005.
2. Guzeldemir E, Toygar HU, Cilasun U. Pain perception and anxiety during scaling in periodontally healthy subjects. J Periodontol. 2008:79(12):2247-55.

3. Maggirias J, Locker D. Psychological factors and perceptions of pain associated with dental treatment. Community Dent Oral Epidemiol. 2002;30(2):151-9.

4. Pihlstrom BL. Measurement of attachment level in clinical trials: probing methods. J Periodontol. 1992;63:1072-7.

5. Wang SF, Leknes KN, Zimmerman GJ, Sigurdsson TJ, Wikesjö UM, Selvig KA. Reproducibility of periodontal probing using a conventional manual and an automated force-controlled electronic probe. J Periodontol. 1995;66(1):38-46.

6. Heins PJ, Karpinia KA, Maruniak JW, Moorhead JE, Gibbs CH. Pain threshold values during periodontal probing: Assessment of maxillary incisor and molar sites. J Periodontol. 1998;69(7):812-8.

7. De Jongh A, Fransen J, Oosterink-Wubbe F, Aartman I. Psychological trauma exposure and trauma symptoms among individuals with high and low levels of dental anxiety. Eur J Oral Sci. 2006;114(4):286-92.

8. Poudyal R, Agrawal P, Shrestha A, Dali M, Bhagat T, Choudhary A. Evaluation of oral hygiene practice, knowledge and attitude among (10-15 Yrs) school children in Dharan, Nepal-a cross-sectional study. Pharmacol Toxicol Biomed Rep. 2015;1(1):14-6.

9. Bhalla A, Singh SB, Sujata CA, Choudhary A. Self-assessment of dental anxiety in patients visiting comprehensive rural health service project. J Depress Anxiety. 2013;2(137):2167-1044.

10. Nepal OHP. National Oral. Health Policy. 2004;1:1-5.

11. Canakci V, Canakci CF. Pain levels in patients during periodontal probing and mechanical non-surgical therapy. Clin Oral Investig. 2007:11(4):377-83.

12. Haefeli M, Elfering A. Pain assessment. Eur Spine J. 2006;15(1):S17-24.

13. Marteau TM, Bekker $\mathrm{H}$. The development of a six-item short-form of the state scale of the Spielberger State-Trait Anxiety Inventory (STAI). Br J Clin Psychol. 1992;31(3):301-6.

14. World Health Organization. Oral health surveys: basic methods. Geneva: World Health Organization; 2013.

15. Sjögren A, Arnrup K, Jensen C, Knutsson IA, Huggare J. Pain and fear in connection to orthodontic extractions of deciduous canines. Int J Paediatr Dent 2010:20(3):193-200.

16. Faisal S, Zehra N, Hussain M, Jaliawala HA, Faisal A. Dental anxiety among patients attending public and private dental hospitals of Karachi. J Pak Dent Assoc. 2015;24(01):46

17. Fardal $\varnothing$, Johannessen AC, Linden GJ. Patient perceptions of periodontal therapy completed in a periodontal practice. J Periodontol. 2002:73(9):1060-6.

18. Liddell A, Locker D. Gender and age differences in attitudes to dental pain and dental control. Community Dent Oral Epidemiol. 1997;25(4):314-8.

19. Shaikh MA, Kamal A. Over dental anxiety problems among university students: perspective from Pakistan. J Coll Phys Surg Pak. 2011;21(4):237-8.

20. Abu-Ghazaleh SB, Rajab LD, Sonbol HN, Aljafari AK, Elkarmi RF, Humphris $G$. The Arabic version of the modified dental anxiety scale. Saudi Med J. 2011:32(7):725-9.

21. Erten $H$, Akarslan ZZ, Bodrumlu E. Dental fear and anxiety levels of patients attending a dental clinic. Quintessence Int. 2006:37(4):304

22. Rachman S, Arntz A. The overprediction and underprediction of pain. Clin Psychol Rev. 1991:11(4):339-55.

\section{Publisher's Note}

Springer Nature remains neutral with regard to jurisdictional claims in published maps and institutional affiliations. 